\title{
A Novel Stabilization Method of the Islanded DC Microgrid
}

\author{
Zhonglin Yang ${ }^{1,2, *}$, Xiaoming $\mathrm{Zha}^{2}$, Leping $\mathrm{Pu}^{2}$ and Xuanfang Yang ${ }^{2}$ \\ ${ }^{1}$ School of Electrical Engineering, Wuhan University, Wuhan 430072, China \\ ${ }^{2}$ School of Electrical Engineering, Naval University of Engineering, Wuhan 430033, China \\ ${ }^{*}$ Corresponding author
}

\begin{abstract}
DC microgrid contains a large number of tightly regulated closed-loop converters, which performance for the constant power loads and may cause stability problem of bus voltage when used as a load since they trend to draw constant power. In order to improve the stability of DC microgrid, this paper proposes a method for stabilization the DC microgrid based on the state feedback. By introducing the state feedback signal, this method increases the damping of the system and ensures the stable operation of the whole DC microgrid. The feasibility and effectiveness of this method are verified by the simulation results.
\end{abstract}

Keywords-DC microgrid; stabilization; islanded mode; constant-power load; buck converter

\section{INTRODUCTION}

In recent years, in order to solve problems of the energy shortage, the environmental pollution and other issues, the distributed power, the microgrid has gained more and more attention and application ${ }^{[1-3]}$. According to the different types of bus voltage, microgrid can be divided into DC microgrid and $\mathrm{AC}$ microgrid. Compared with the $\mathrm{AC}$ microgrid, DC microgrid has the advantages of simple control, high efficiency and high reliability, so it is gradually being increasingly concerned about ${ }^{[4-8]}$. However, the DC microgrid contains a large number of load converters, whose characteristics are constant power loads and may cause instability of the DC micro grid bus voltage ${ }^{[9-13]}$.

Some of the existing papers analyze the stability of the DC microgrid and introduce some methods to improve the stability of DC microgrid. In paper [14], the equivalent impedance of the converter which was connected to the Grid was changed by increasing the active damping signal, which can improve the stability of the DC microgrid. However, this method only can be used for microgrid in grid connected conditions. A method for improving the stability of DC microgrid by introducing the virtual capacitor into the load point is proposed [15], but the method is only suitable for the case of single load the single load. In this paper, the state feedback technique was introduced to improve the stability of the DC microgrid. The method is simple and convenient to implement.

\section{SySTEM MODELING AND STABILITY ANALYSIS}

Typical DC microgrid structures are shown in Figure I, which contains a large number of power electronic converters.
One of the $\mathrm{AC} / \mathrm{DC}$ or $\mathrm{DC} / \mathrm{DC}$ source converters connects with DC microgrid and maintains the bus voltage. When the load point converters work in the constant voltage mode and the control performance is good, the load point converters performance the constant power loads (CPLs) to the DC microgrid [16]. And in ordinary climatic conditions, in order to make full use of renewable energy, the PV, wind power and other distributed power generally work at the maximum power tracking (MPPT) mode. At this time they can be regarded as constant power source [6].

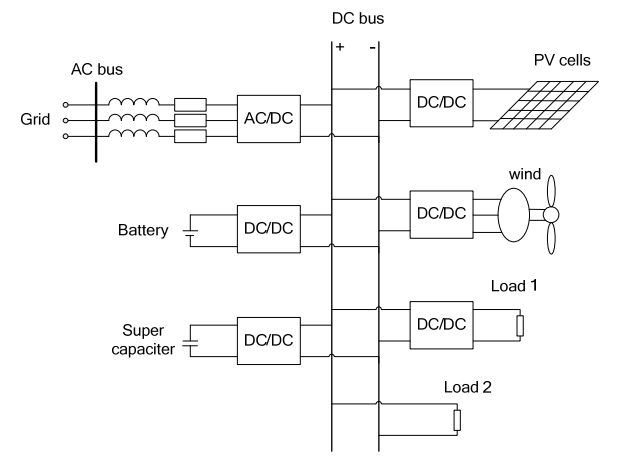

FIGURE I. SCHEMATIC DIAGRAM OF A DC MICROGRID

When the AC large power grid fails, the source side $\mathrm{AC} / \mathrm{DC}$ converter stops working and the DC microgrid runs in the isolated mode. At this time, a distributed power source maintains the DC bus voltage through the DC/DC converter.

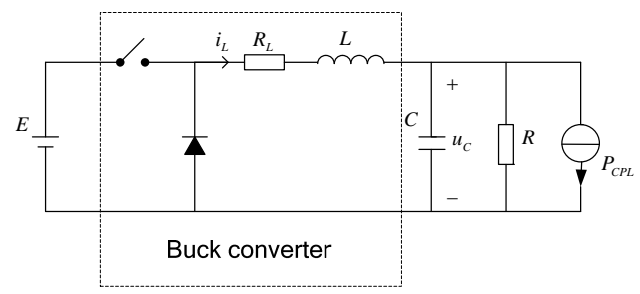

FIGURE II. THE SIMPLED MODEL OF A DC MICROGRID WHICH SUPPORTS BUS VOLTAGE BY THE BUCK CONVERTER

To analyze simply, the case in which the Buck converter was used to maintain the DC microgrid bus voltage was taken as the example to study system stability. The simplified model of the DC microgrid is shown in Figure II. Where E is the input voltage of Buck converter, $\mathrm{L}$ is the filter inductor of the Buck converter, $\mathrm{C}$ is the equivalent capacitance of all the capacitances which connect to the $\mathrm{DC}$ bus, $\mathrm{R}$ is equivalent 
resistance of all the resistant load, and the PCPL is the equivalent constant power load, which value is the sum of the input power of all the constant power loads subtract the sum of all the output power of the constant power sources.

By using the state average method, the circuit equations can be written as

$$
\left\{\begin{array}{l}
L \frac{d i_{L}}{d t}=E \cdot d-u_{C} \\
C \frac{d u_{C}}{d t}=i_{L}-\frac{u_{C}}{R}-\frac{P_{C P L}}{u_{C}}
\end{array}\right.
$$

Where $d$ is the duty cycle of the Buck converter, and in the open loop control mode it is a constant. The equilibrium point of the system can be calculated by the following equations

$$
\begin{gathered}
U_{C}=E \cdot d \\
I_{L}=\frac{U_{C}}{R}+\frac{P_{C P L}}{U_{C}}
\end{gathered}
$$

By linearization of equations (1) at its equilibrium point, the characteristic equation of the system is calculated to be

$$
H(s)=s^{2}+\frac{1}{C}\left(\frac{1}{R}-\frac{P_{C P L}}{U_{C}^{2}}\right)+\frac{1}{L C}
$$

Applying the Routh-Hurwitz stability criterion, the system stable condition can be deduced which was

$$
P_{C P L}<\frac{U_{C}{ }^{2}}{R}
$$

From the equation (5), it can be seen that in order to make the DC microgrid stability, the power of CPLs must be less than the power of resistive loads. But In the actual DC microgrid, the loads usually include $75 \%-80 \%$ of the CPLs and $20-25 \%$ of the resistance loads [14]. Therefore usually, the system is not stable, and some technique needs to be used to improve the system stability.

\section{The Stabilization Method FOR ISLANDED DC Microgrid BASED ON THE STATE FEEDBACK}

From (5), it can be known that the negative incremental impedance characteristic of constant power loads reduces the system's damping, which leads to the instability of the system. In order to increase the damping of the system and ensure the stability of the system, the state feedback signal can be introduced in the duty cycle signal, which makes the duty cycle signal as follows

$$
d=\frac{1}{E}\left[U_{C}-k_{1} \frac{d u_{C}}{d t}-k_{2}\left(u_{C}-U_{C}\right)\right]
$$

From (6) it is known that the introduction of the state feedback signal does not change the equilibrium point of the system. Considering (1) and (6), the circuit equations can be written in the form of

$$
\left\{\begin{array}{l}
L \frac{d i_{L}}{d t}=-\frac{k_{1}}{C} i_{L}+\left(\frac{k_{1}}{R C}-k_{2}-1\right) u_{C}+\frac{k_{1} P_{C P L}}{C u_{C}}+\left(k_{2}+1\right) U_{C} \\
C \frac{d u_{C}}{d t}=i_{L}-\frac{u_{C}}{R}-\frac{P_{C P L}}{u_{C}}
\end{array}\right.
$$

From (7), the characteristic equation of the DC microgrid is calculated to be

$$
H_{1}(s)=s^{2}+\frac{1}{C}\left(\frac{k_{1}}{L}+\frac{1}{R}-\frac{P_{C P L}}{U_{C}^{2}}\right)+\frac{k_{2}+1}{L C}
$$

The roots of (8), which are the poles of the system, can be analyzed using the Routh-Hurwitz method. Such an analysis shows that if (9) are achieved, the DC microgrid is stable.

$$
\left\{\begin{array}{l}
k_{1}>\left(\frac{P_{C P L}}{U_{C}^{2}}-\frac{1}{R}\right) L \\
k_{2}>-1
\end{array}\right.
$$

\section{The SimUlation EXPERIMENT}

To verify the effectiveness for the proposed method, the simulation model was built by Matlab/Simulink, which structure was shown in Figure III.

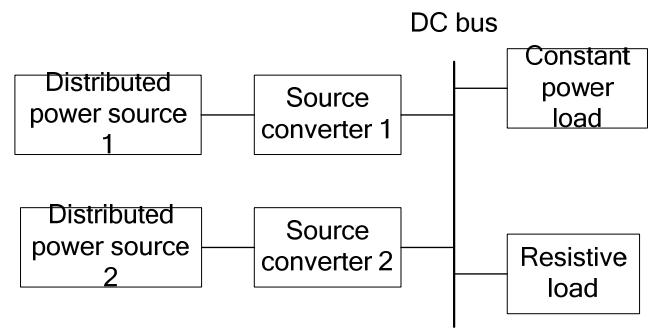

FIGURE III. THE DC MICROGRID WITH TWO GENERATORS AND TWO LOADS

The source converter 1 was the Buck converter which maintains the DC bus voltage. The source converter 2 was the converter in the constant power control. The parameters of simulation system are as follows: $\mathrm{E}=400 \mathrm{~V}, \mathrm{~d}=0.5, \mathrm{~L}=8 \mathrm{mH}$, $\mathrm{C}=0.5 \mathrm{mF}$. The output power of the constant power source was $500 \mathrm{~W}$, and the power of CPL was $2500 \mathrm{~W}$. So the value of the equivalent constant power load PCPL was $2000 \mathrm{~W}$. The value of resistive load R was $40 \Omega$. The switching frequency of the converter was $10 \mathrm{kHz}$. Simulation results are shown in the Figure IV and Figure V. 
When the system did not join the state feedback signal, the eigenvalues at the equilibrium point $(200 \mathrm{~V}, 15 \mathrm{~A})$ are $25 \pm \mathrm{j} 499.37$ by calculation from (4). The bus voltage $\mathrm{uC}$ and the buck converter inductor current iL were shown in Figure IV. Because the real part of the eigenvalues are greater than zero, the bus voltage $\mathrm{uC}$ and inductor current iL diverge. Until the inductor current decreased to zero, the bus voltage maintains greatly oscillation.

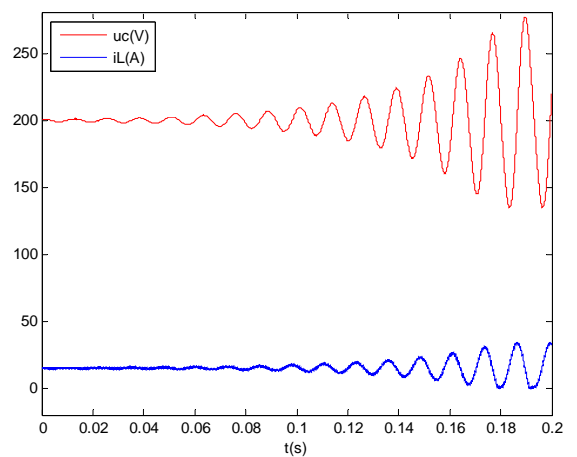

FIGURE IV. THE BUS VOLTAGE AND INDUCTANCE CURRENT OF DC MICROGRID SUPPORTED BY BUCK CONVERTER WITHOUT THE STATE FEEDBACK SIGNAL

When the state feedback signal is added, The DC bus voltage $\mathrm{uC}$ and the inductor current iL are shown in Figure V. In order to satisfy (9), it can be selected that $\mathrm{k} 1=0.005$ and $\mathrm{k} 2=1$. From (8), the eigenvalues of the system at the equilibrium point $(200 \mathrm{~V}, 15 \mathrm{~A})$ are $-37.50 \pm \mathrm{j} 706.11$. As the real parts of eigenvalues are less than zero, the system is stable.

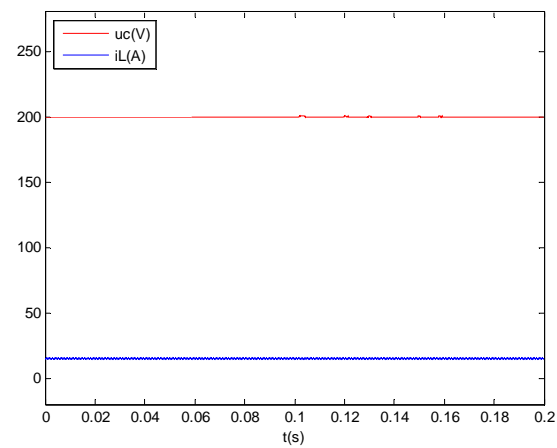

FIGURE V. THE BUS VOLTAGE AND INDUCTANCE CURRENT OF DC MICROGRID SUPPORTED BY BUCK CONVERTER WITH THE STATE FEEDBACK SIGNAL

As seen from the Figure V, it can be shows that adding the state feedback signal can enlarge the system's damping and counteract the adverse effect of the constant power load, which make sure the stability of the DC microgrid.

\section{THE CONCLUSION}

In aim to the stability problem of the DC microgrid which was caused by the CPLs, the state feedback technique was introduced. This technique was used to counteract the destabilizing effect of the CPLs by adding the state feedback signal to the duty cycle of the source converters, so as to realize the stability of DC microgrid. This method only need use the signal of DC bus voltage, and the realization is simple. The validity of the proposed method has been verified by simulation experiments.

\section{REFERENCES}

[1] Hiroaki Kakigano, Yushi Miura, Toshifumi Ise. Distribution voltage control for DC microgrids using fuzzy control and gain-scheduling technique[J], IEEE Transaction on Power Electronics, 2013, 28(5):22462257.

[2] Sandeep Anaad, B. G. Fernandes. Reduced-order model and stability analysis of low-voltage DC microgrid[J], IEEE Transaction on Industrial Electronics, 2013, 60(11):5040-5049.

[3] Hiroaki Kakigano, Yushi Miura, Toshifumi Ise. Low-voltage bipolartype DC microgrid for super high quality distribution[J], IEEE Transaction on Power Electronics, 2010, 25(12):3066-3075.

[4] Sandeep Anand, Baylon G. Fernandes, Josep M. Guerrero. Distributed control to ensure proportional load sharing and improve voltage regulation in low-voltage DC microgrids[J], IEEE Transaction on Power Electronics, 2013, 28(4):1900-1913.

[5] Sijo Augustine, Mahesh K. Mishra, N. Lakshminarasamma. Adaptive droop control strategy for load sharing and circulating current minimization in low-voltage standalone DC microgrid[J], IEEE Transactions on Sustainable Energy, 2015, 6(1):132-141

[6] Andre Pires Nobrega Tahim, Daniel J. Pagano, Eduardo Lenz, etc. Modeling and stability analysis of islanded DC microgrids under droop control[J], IEEE Transaction on Power Electronics, 2015, 30(8):45974607.

[7] Vahidreza Nasirian, Seyedali Moayedi, Ali Davoudi, etc. Distributed cooperactive control of DC microgrids[J], IEEE Transaction on Power Electronics, 2015, 30(4):2288-2303.

[8] Alexia Kwasinski, Chimaobi N. Onwuchekwa. Dynamic behavior and stabilization of DC microgrids with instantaneous constant-power loads[J]. IEEE Transation on Power Electronics, 2011, 26(3):822-834.

[9] Weijing Du, Junming Zhang, Yang Zhang. Stability criterion for cascaded system with constant power load[J], IEEE Transactions on Power Electronics, 2013, 28(4): 1843-1851.

[10] Didier Marx, Pierre Magne, Babak Nahid-Mobarakeh. Large signal stability analysis tools in DC power systems with constant power loads and variable power loads-a review[J], IEEE Transactions on Power Electronics. 2012, 27(4): 1773-1787.

[11] E. Barklund, N. Pogaku, M. Prodanvic, ect. Energy management in autonomous microgrid using stability-constrained droop control of inverters[J], IEEE Transactions on Power Electronics. 2007, 23(5): 2346-2352.

[12] Y. A. -R. I. Mohamed, E. F. Ei-Saadany. Adaptive decentralized droop controller to preserve power sharing stability of paralleled inverters in distributed generation[J], IEEE Transactions on Power Electronics. 2008, 23(6): 2806-2816.

[13] N. Pogaku, M. Prodanovic T. C. Green. Modeling, analysis and testing of autonomous operation of an inverter-based microgrid[J], IEEE Transactions on Power Electronics. 2007, 22(5): 613-625.

[14] Amr Ahmed A. Radwan, Yasser Abdel-Rady I. Mohamed. Linear active stabilization of converter-dominated DC microgrid[J]. IEEE Transaction on Smart Grid, 2012, 3(1):203-216.

[15] Pierre Magne, Didier Marx, Babak Nahid-Mobarakeh. Large-signal stabilization of a DC-link supplying a constant power load using a virtual capacitor: Impact on the Domain of Attraction[J].IEEE Transactions on Industry Applications, 2012,48( 3):878-887.

[16] Jiabin Wang, David Howe. A power stabilizing control strategy for DC power systems with constant power loads, IEEE Transactions on Power Electronics. 2008, 23(6): 2986-2989.. 